\title{
Block Matching Algorithm for Motion Estimation using Previous Motion Vector Pattern
}

\author{
Neha Singh \\ Department of Computer \\ Science \& Engineering \\ Pranveer Singh \\ Institute of Technology, Kanpur \\ Dr. A P J Abdul Kalam \\ Technical, University \\ Lucknow, India
}

\author{
Awanish Mishra \\ Department of Computer \\ Science \& Engineering, \\ Pranveer Singh \\ Institute of Technology, Kanpur \\ Dr.A P J Abdul Kalam \\ Technical, University \\ Lucknow, India
}

\begin{abstract}
Correct motion estimation is major concern in developing video coding applications. Among various available motion estimation techniques block matching is most favorites because of its effectiveness and simplicity for hardware and software both. In block based motion estimation techniques, computation is reduced by limiting the number of candidate search points within the search window or by implementing effective search criterion for picking the correct block. Previous pattern based motion estimation algorithm has been suggested in literature which controls the number of search points drastically and hence reduce the computation cost. In this manuscript, a new block based searching method is proposed that uses the motion vector for the just before calculated block as the motion vector for the current block and directly map the block in referenced frame accordingly. Experimental results show that an increase up to $15 \%$ in terms of peak signal to noise ratio (PSNR) has been achieved than conventional block matching algorithm with almost same execution time. Further, in terms of quality/computation ratio where quality and computation has been measured in terms of PSNR and execution time respectively, proposed method has $10-20 \%$ gain over existing block based technique.
\end{abstract}

\section{General Terms}

Motion vector, motion estimation algorithm, Block matching algorithms.

\section{Keywords}

Reference frame, motion estimation, mean absolute error, block matching method, video coding.

\section{INTRODUCTION}

In video data, both type of redundancy is present. These redundancies are spatial and temporal. These redundancies can be reduced by video compression techniques. For removing large amount of redundancies from the video data, inter frame predicted coding is defined.

The main purpose of conventional predictive coding is to code and transmit the difference between the current frame and the predicted frame (based on previous frame). With the help of encoding side, motion can be estimated in the current frame with respect to previous frame. Then a motion compensated image for the current frame is created which is built of blocks of image from the previous frame. The motion vectors for blocks are transmitted, which is used for motion estimation. And the difference of the compensated image with the current frame is encoded and sent. Using encoder, the encoded image is encoded and than it can be used as a reference frame for the subsequent frame. Decoder can reverse the process and create a full frame. In video compression, the main aim of using motion estimation is to reduce the number of bits by sending JPEG encoded difference images, which inherently have less energy and can be highly compressed as compared to sending a full frame that is JPEG encoded.

First frame should always sent full and in similar way some other frames that might occur at some regular interval(like every $6^{\text {th }}$ frame) the standards of video is not specify at may be changed as per dynamics of video.

Motion estimation required more computation and resources for entire compression process. Due to such high computation cost this field has gone through a tremendous research activity. In this paper various fundamental block matching algorithm has been implemented and evaluated using different search criterion.

To reduce error from the video, prediction must be good. So that bit rate can be reduced during transmission, if a scene is still. Good prediction in the current frame for a particular pixel is the same pixel in the previous frame and the error is zero, for still scene in a video sequence, whereas, in a motion sequence, a good prediction for the current pixel is same pixel in the moving better. Motion estimation is the process of finding motion vectors in order to estimate the modification of images with the help of the camera or the object present in the image the motion vectors are applied and it is called as motion compensation. The main element for video compression is $\mathrm{ME}$ and motion compensation,

For inter frame predictive coding large amount of motion compensation algorithm has been defined. In this paper only block matching algorithm has been used, the main purpose of these algorithm is to estimate the motion in a block by block basis, that is blocks in the current frame, a block from the previous frame is found ,that is said to match this block based certain criterion.

Exhaustive search or full search is one of the first algorithms which are used for block based motion compensation algorithm. In this algorithm each block is searched within a given search window and then compared with the current block.

If the best match found the search will stop there, although this algorithm provide better quality of predicted image, but it is computationally expensive. So various efficient block based algorithm have been defined. Such as full search [FS] Three Step Search [3] (TSS), New TSS [4], Two Dimensional Logarithmic Search [2] (TDL), Four Step Search [5] (FSS), Gradient Descent Search [6], Diamond Search [7, 8] Hexagon based Search $[9,10]$. 


\section{BLOCK MATCHING ALGORITHM}

The main idea behind the motion estimation is that the pattern corresponding to object and background of the video sequence move within the frame to frame corresponding object in the subsequent frame.

The main purpose of block matching algorithm is to divide the current frame into various macro blocks and then these macro blocks compared with corresponding blocks and its adjacent neighbors in the previous frame to create a vector, that define the displacement between the macro blocks in the previous frame. This movement for all the macro blocks consists of comprising a frame. Constitutes the motion estimated in the current frame. The search location is constraints up to ' $\mathrm{P}$ ' pixels in all sides of the macro block in the frame to catch the best match where $\mathrm{P}$ is known as location of search parameters. Larger motion movement needs a large value of $\mathrm{P}$ and the larger the search parameter the more computationally expensive process becomes. Normally, the macro block is taken as a square of $16 \times 16$ pixels. And the search parameter ' $p$ ' is 7 pixels. The matching of one macro blocks with another is depends on the output of cost function. The macro block that result in the least cost is the one that matches the closest to current block.

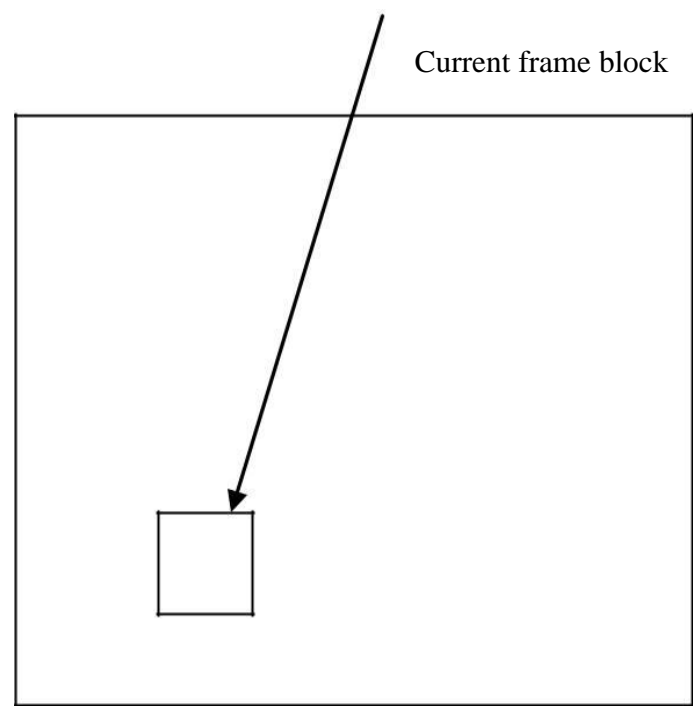

Current frame
Location of the current block

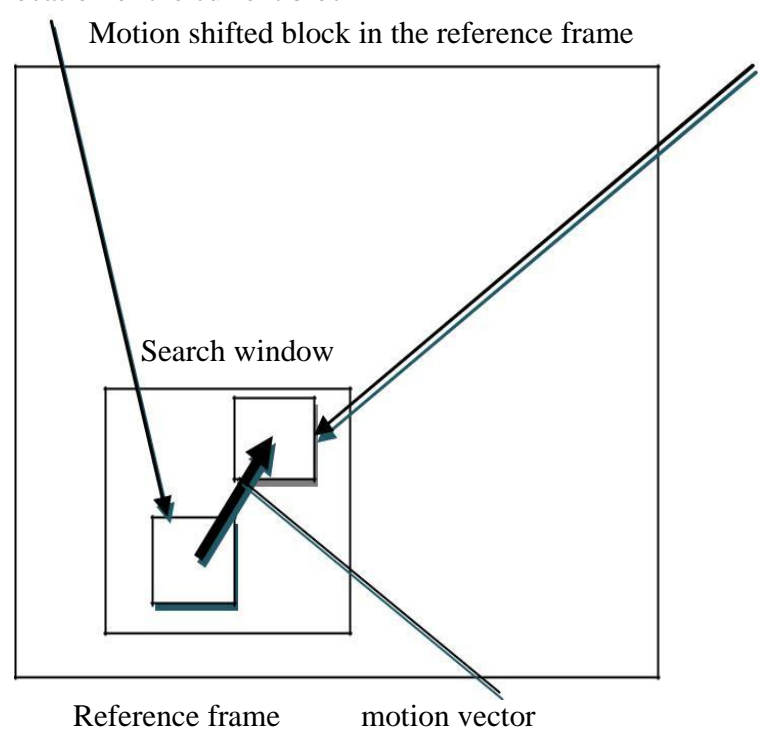

Fig1 .Block based motion estimation

\section{PROPOSED METHOD}

In the proposed method for the motion estimation using block matching techniques for every block from the current frame is initially mapped with the motion vector obtained for the previous block in the reference frame. If the criterion for the correct matching block is not satisfied in this way then only conventional block matching technique should be used to find motion vector for the matching block. Idea for this arrangement to find the faster and accurate motion vector is that if the one block has been search in reference frame then other neighboring macro blocks will also have the same motion vector because, every macro block belonging to the same object will definitely have the same motion vector. It is not always true that neighboring macro blocks belongs to the same objects fully and hence proposed method is also using search methodology. For the first block from the current frame where the need is to find motion vector for matching block, this method will not be applicable because at this time no motion vector is available. Following are the steps for proposed blocked based searching method:

1. for the first block from the current frame use conventional method to find motion vector to match with a block in the reference frame.

2. Next onwards for every block use the previous motion vector to match with the block from the reference frame.

3. If the step-2 does not provide correct matching block then use the conventional method to find motion vector to match with a block in the reference frame.

4. Use step-2 for every block from the current frame to find matching block. 


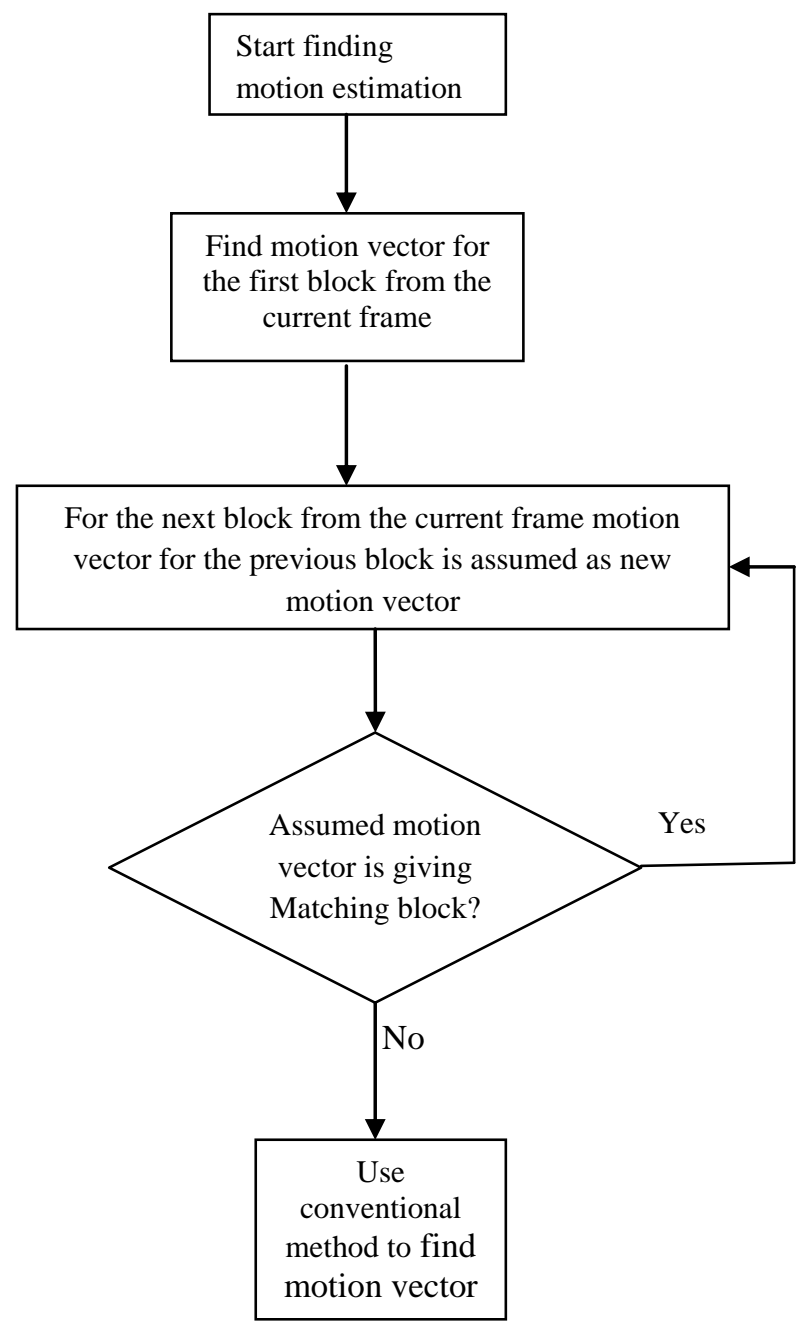

Fig2.Flow chart of proposed algorithm

\section{EXPERIMENTAL RESULT}

Performance evaluation of the proposed block based matching method to find correct matching block in the reference frame for a block from the current frame using the estimation of motion vector has been carried out with respect to the Mean absolute difference(MAD) in terms of two parameters average PSNR and execution time (seconds). Three step search technique has been used as conventional method for motion estimation and nine video sequences with different features have been taken as input. Search window size used for motion estimation is \pm 15 and size of each block is $16 \times 16$. Further, four sub-blocks representation of a block has been used for comparison purposes. All simulation work has been carried out in MATLAB R2013.

Table 1 shows the performance comparison of proposed method in terms of PSNR for frame distance one and it is very clear that it has better results in comparison to three step search and new three step search respectively whereas Table 2 shows the same for frame distance two and the proposed criterion has slightly more PSNR gain for TSS and NTSS respectively.
Table 1: Performance comparison in terms of PSNR for frame distance 1

\begin{tabular}{|l|c|c|c|}
\hline Video inputs & TSS & NTSS & $\begin{array}{c}\text { Proposed } \\
\text { Method }\end{array}$ \\
\hline $\begin{array}{l}\text { Cactus comb } \\
(240 x 352)\end{array}$ & 13.6 & 18.6 & 24.55 \\
\hline Car phone (144x176) & 16.5 & 17.9 & 24.81 \\
\hline Flower (240x352) & 12.2 & 15.4 & 21.32 \\
\hline $\begin{array}{l}\text { Miss America } \\
\text { (144x176) }\end{array}$ & 24.6 & 30.6 & 32.45 \\
\hline Tsunami (240x320) & 18.7 & 22.5 & 26.35 \\
\hline Trojan lift (240x320) & 12.8 & 16.4 & 20.16 \\
\hline India pride (240x320) & 13.9 & 17.3 & 22.58 \\
\hline Susie (240x352) & 26.2 & 34.2 & 36.56 \\
\hline Airbus (288x352) & 22.6 & 27.6 & 29.52 \\
\hline
\end{tabular}

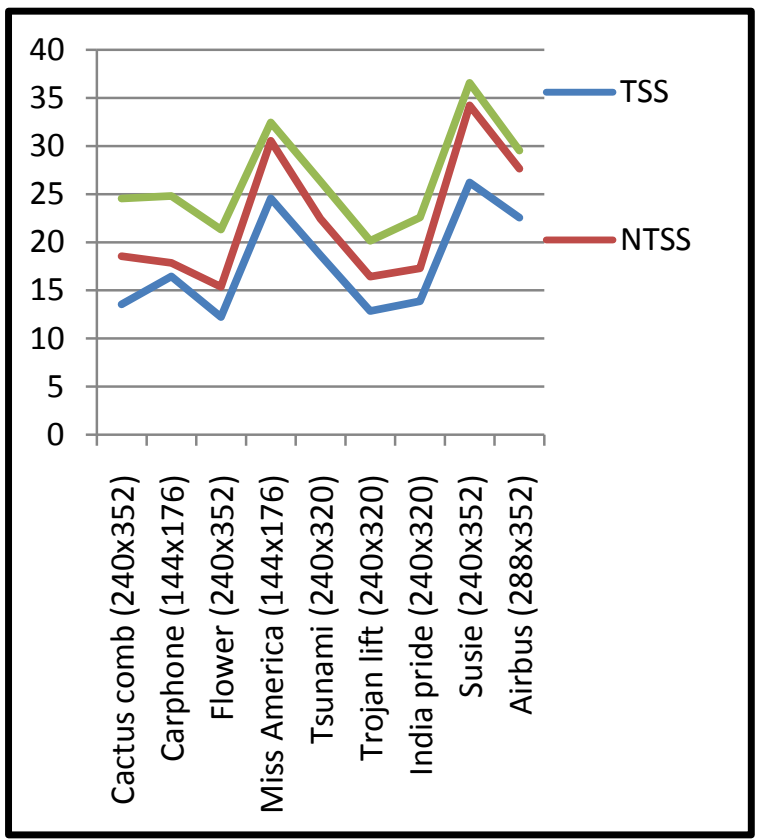

Fig3.Graph representation of performance comparison in terms of PSNR for frame distance 1

In the above figure various videos have been taken as input for calculating the PSNR value for frame distance one. The graphical representation has been shown in the Fig3.From the graph it is clearly showing the performance comparison of proposed algorithm in terms of PSNR for frame distance 1.

Table 2: Performance comparison in terms of PSNR for frame distance 2

\begin{tabular}{|l|c|c|c|}
\hline Video inputs & TSS & NTSS & $\begin{array}{c}\text { Proposed } \\
\text { Method }\end{array}$ \\
\hline $\begin{array}{l}\text { Cactus comb } \\
(240 \times 352)\end{array}$ & 11.34 & 13.45 & 15.44 \\
\hline $\begin{array}{l}\text { Car phone } \\
(144 x 176)\end{array}$ & 13.45 & 12.34 & 14.45 \\
\hline Flower (240x352) & 11.43 & 14.87 & 18.54 \\
\hline $\begin{array}{l}\text { Miss America } \\
(144 x 176)\end{array}$ & 20.56 & 22.65 & 27.54 \\
\hline Tsunami (240x320) & 16.34 & 16.98 & 18.34 \\
\hline
\end{tabular}




\begin{tabular}{|l|c|c|c|}
$\begin{array}{l}\text { Trojan lift } \\
(240 \times 320)\end{array}$ & 11.64 & 13.65 & 18.98 \\
\hline $\begin{array}{l}\text { India pride } \\
(240 \times 320)\end{array}$ & 11.83 & 14.86 & 18.45 \\
\hline Susie (240x352) & 22.21 & 26.65 & 31.45 \\
\hline Airbus(288x352) & 20.52 & 21.89 & 27.22 \\
\hline
\end{tabular}

Fig4 shows the graph representation of performance comparison in terms of PSNR for frame distance 2.

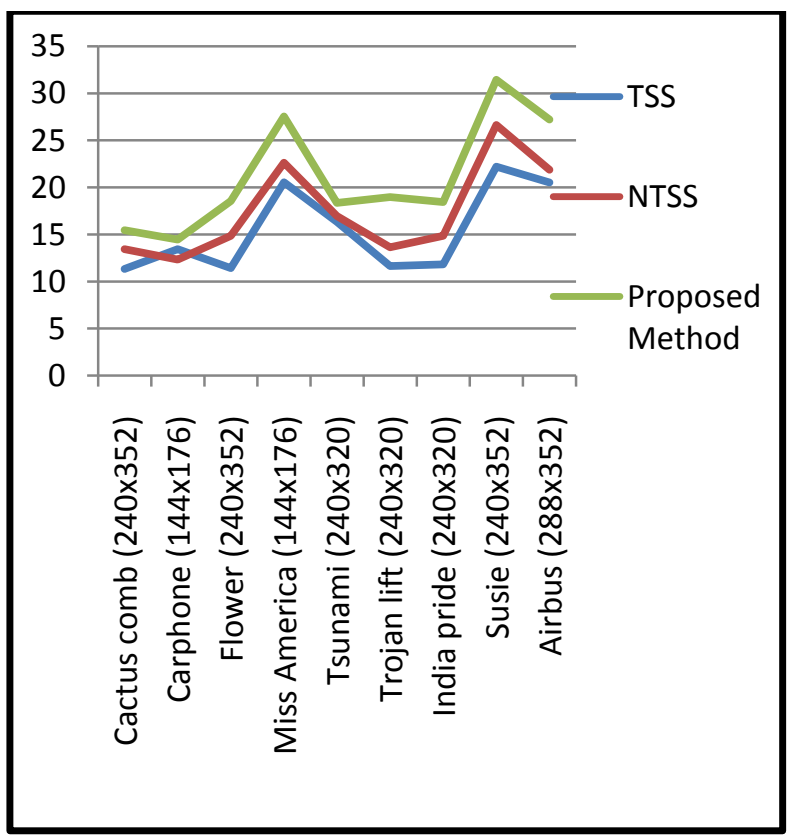

Fig4.Graph Representation of performance comparison in terms of PSNR for frame distance 2

Table 3: Performance comparison in terms of execution time (sec.) for frame distance 1

\begin{tabular}{|l|c|c|c|}
\hline Video inputs & TSS & NTSS & $\begin{array}{c}\text { Proposed } \\
\text { Method }\end{array}$ \\
\hline $\begin{array}{l}\text { Cactus comb } \\
(240 x 352)\end{array}$ & 12.853 & 10.043 & 8.908 \\
\hline $\begin{array}{l}\text { Car phone } \\
(144 \times 176)\end{array}$ & 12.843 & 11.872 & 9.563 \\
\hline Flower (240x352) & 12.754 & 9.452 & 8.086 \\
\hline $\begin{array}{l}\text { Miss America } \\
(144 x 176)\end{array}$ & 12.808 & 8.765 & 7.456 \\
\hline Tsunami (240x320) & 12.901 & 10.012 & 8.456 \\
\hline $\begin{array}{l}\text { Trojan lift } \\
(240 x 320)\end{array}$ & 12.857 & 9.101 & 6.657 \\
\hline $\begin{array}{l}\text { India pride } \\
(240 x 320)\end{array}$ & 12.788 & 11.181 & 10.073 \\
\hline Susie (240x352) & 12.805 & 9.006 & 7.658 \\
\hline Airbus (288x352) & 12.788 & 10.248 & 7.006 \\
\hline
\end{tabular}

Fig 5 shows the graph representation of performance comparison in terms of execution time (sec.) for frame distance 1 .

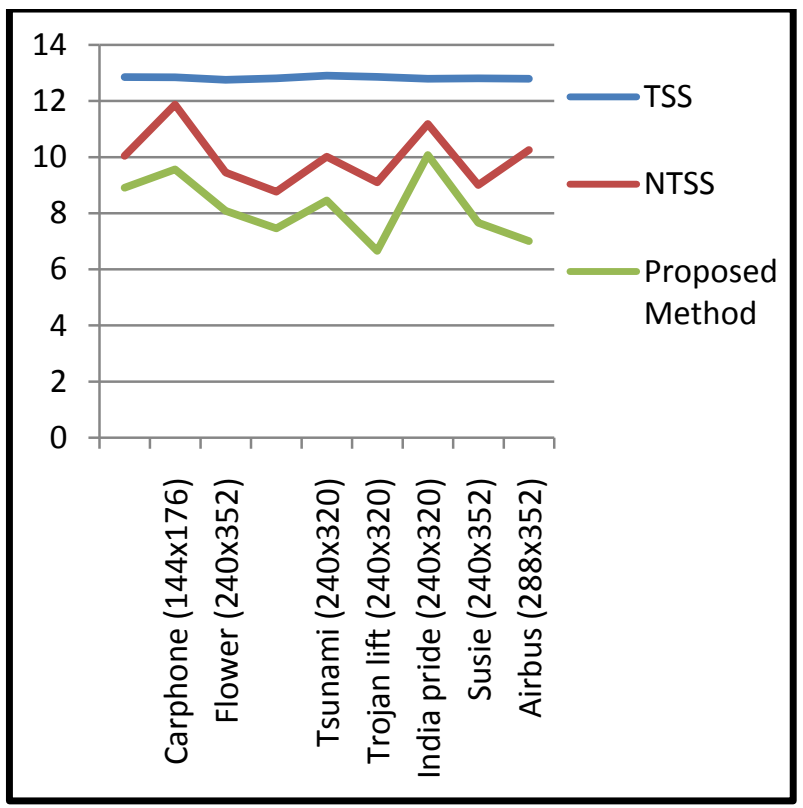

Fig 5.Graph representation of performance comparison in terms of execution time (sec.) for frame distance 1

Table 4: Performance comparison in terms of execution time (sec.) for frame distance 2

\begin{tabular}{|l|l|l|l|}
\hline Video inputs & TSS & NTSS & $\begin{array}{l}\text { Proposed } \\
\text { Method }\end{array}$ \\
\hline $\begin{array}{l}\text { Cactus comb } \\
(240 x 352)\end{array}$ & 12.545 & 11.534 & 9.405 \\
\hline $\begin{array}{l}\text { Car phone } \\
(144 x 176)\end{array}$ & 12.564 & 11.765 & 10.365 \\
\hline Flower (240x352) & 12.765 & 10.342 & 8.001 \\
\hline $\begin{array}{l}\text { Miss America } \\
(144 x 176)\end{array}$ & 12.528 & 10.235 & 8.236 \\
\hline $\begin{array}{l}\text { Tsunami lift } \\
(240 x 320)\end{array}$ & 12.438 & 10.124 & 9.235 \\
\hline $\begin{array}{l}\text { Trojan pride } \\
(240 x 320)\end{array}$ & 12.005 & 11.295 & 10.984 \\
\hline $\begin{array}{l}\text { India } \\
(240 x 320)\end{array}$ & 12.876 & 10.436 & 9.203 \\
\hline Susie (240x352) & 12.129 & 11.984 & 9.164 \\
\hline Airbus (288x352) & & 8.825 \\
\hline
\end{tabular}




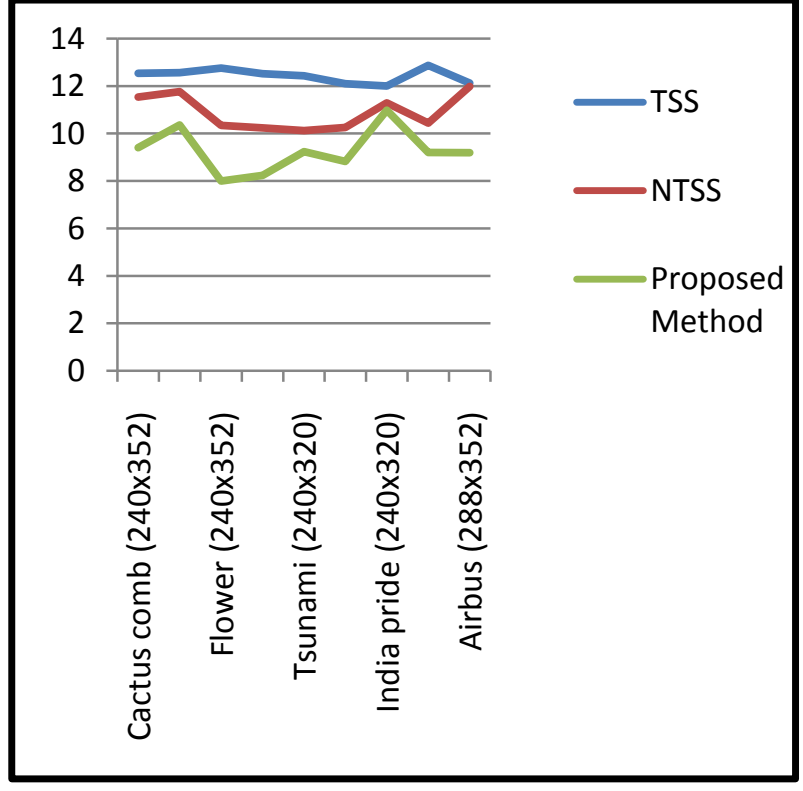

Fig6. Graph representation of Performance comparison In terms of execution time (sec.) for frame distance 2

\section{CONCLUSIONS}

In this manuscript, a two level block matching algorithm for motion estimation has been suggested. The proposed method uses previous motion vector to find the matching block in reference frame. This proposed method for block based searching is showing very motivating results in terms of PSNR and computations (execution time) when used with three step search and new three step search.

\section{REFERENCES}

[1] T.Sikora, "MPEG Digital Video Coding Standards," IEEE Signal Processing Magazine , pp. 82-100, September 1997.

[2] J R Jain and A K Jain, "Displacement Measurement and its application in Interframe Coding, "IEEETransactions on Communications, vol. 29, no. 12, December 1981.

[3] k.Iinuma, A.Hirano and Y.Ishiguro T.Koga, "MotionCompensated Interframe Coding for Video Conferencing," in Proc NTC81, New Orleans,LA, 1981.

[4] B.Zeng and M.L.Liou R.Li, "A New Three Step Search Algorithm For Block Motion Estimation," IEEE Transactions on Circuits and Systems for Video Technology, vol. 4, no. 4, August 1994.

[5] L.Man Po and W.C.Ma, "A Novel Four Step Search Algorithm for Fast Block Motion Estimation," IEEE Ttransactions on Circuits and Systems for Video Technology, vol. 6, no. 3, June 1996.

[6] L.Kuo and E.Feig, "A Block Based Gradient Descent Search Algorithm for Block motion Estimation in Video Coding," IEEE Transactions on Circuits and Systems for video Technology, vol. 6, no. 12, August 1996.

[7] S.Zhu and K.K.Ma, "A New Diamond Search Algorithm for Block Matching Motion estimation," IEEE Transactions on image processing, vol. 9, no. 2, February 2000.

[8] X.Lin, L. Chau and L.M.Po C.Zhu, "Enhanced Hexagonal Search for Fast Block Motion Estimation,"
IEEE transactions on circuits and systems for Video Technology, vol. 14, no. 10, October 2004.

[9] Manojit Panda and Swaplila S.Dash Santosh kuchhotary, "An Efficient three step algorithm for fast motion estimation in mobile domain," International Journal of Computer And Communication Technology, vol. 3, pp. 109-112, 2014.

[10] Aroh Barjatya, "Block Matching Algorithms For Estimation," Member IEEE, 2004.

[11] Bing Zeng and ming L.Liou Renxiang Li, "A new three step search algorithm for block matching motion estimation," IEEE Transaction Circuits and Systems for Video Technology, vol. 4, pp. 438-442, August 1994.

[12] D.V Manjunathan and Sianarayan, "Comparison and implementation of Fast block matching motion estimation algorithms for video compression," International Journal of Engineering Science and Technology, vol. 3, no. 10, October 2011.

[13] Hussain Abu Surrah And Mohd. Junedul Haque, "A Comparative approach for block matching algorithms used for motion estimation," International Journal of Computer science Issues, vol. 11, no. 2, May 2014.

[14] Dr. Rajeev pandey Gaurav vijayvargiya Dr. Sanjay Silakari, "A survey on various techniques of image compression," (IJCSIS)International Journal of Computer Science And information Security, vol. 11, no. 10, October 2013.

[15] Chen-Han Tsai,Chun-Fu Shen And Liang-Gee Chen YuWen Huang Ching-Yen chen, "Survey on block matching motion estimation algorithms and architectures with new results," Journal of VLSI Signal Processing, vol. 42, pp. 297-320, February 2006.

[16] wei-Yi wei, "An Introduction to image Compression," in National Taiwan University,Taipei, Taiwan,Roc.

[17] Iain E.G.Richardson, Video Codec Design.: John Wiley \& Sons Ltd, 2002.

[18] Dr.Saroj Choudhary Pandey, "literature review on block matching motion estimation algorithm for video compression," International Journal of Advanced Research In Computer Engineering \& Technology, vol. 4, no. 5, May 2015.

[19] S.Metkar and S.Talbar, "Performance Evaluation of Block Mtching Algorithms For Video Coding," in Motion Estimation Techniques for Digital Video Coding., 2013, ch. 2.

[20] Y.Nie and K.K.Ma, "Adaptive rood pattern search for fast block block matching motion estimation," IEEE Transaction Image Processing, vol. 11, p. 1442_1448, 2002. 